Pacific Journal of Mathematic 


\section{A SHAPE FIBRATION WITH FIBERS OF DIFFERENT SHAPE}

\section{James Keesling AND Sibe Mardeší́*}

Let $E$ and $B$ be metric continua. Let $p: E \rightarrow B$ be a shape fibration in the sense of Mardešić and Rushing. If $B$ is arcwise connected, then all the fibers of $p$ have the same shape. This is also true if $B$ is connected by shape paths in the sense of Krasinkiewicz and Minc. It had been asked whether that this would be true without any assumptions other than $B$ being a continuum. In this paper an example is given of a shape fibration $p: E \rightarrow B$ with $E$ and $B$ metric continua such that $p$ has fibers of different shape.

1. Introductions. Let $\underline{E}=\left(E_{n}, h_{n}\right)$ and $\underline{B}=\left(B_{n}, q_{n}\right)$ be inverse sequences of metric compacta. A level map $\underline{p}: \underline{E} \rightarrow \underline{B}$ is a sequence of maps $p_{n}: E_{n} \rightarrow B_{n}$ such that for each $n$ the following diagram commutes.

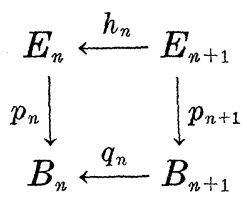

If each $p_{n}$ is a fibration, we say that $\underline{p}$ is a level map of fibrations. Each level map $\underline{p}: \underline{E} \rightarrow \underline{B}$ induces a map $p: E \rightarrow B$ between the inverse limits $E=\lim \underline{E}$ and $B=\lim \underline{B}$.

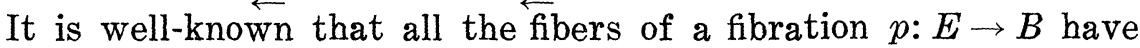
the same homotopy type provided $B$ is pathwise connected. If $p: E \rightarrow B$ is induced by a level map of fibrations (or more generally by a shape fibration in the sense of $\mathrm{S}$. Mardešic and T. B. Rushing ([8] and [7])), and if $B$ is pathwise connected, then any two fibers have the same shape. This is actually true more generally when $B$ is connected by shape paths in the sense of J. Krasinkiewicz and P. Minc [6]. In this paper we show that one cannot strengthen this by assuming only that $B$ is connected. More precisely we prove the following theorem.

THeOREM 1. There exist continua $E$ and $B$ and $a$ map $p: E \rightarrow B$ induced by a level map of fibrations $\left\{p_{n}\right\}$ such that $p$ admits two fibers of different shape.

From the construction proving Theorem 1 we actually have that each $p_{n}: E_{n} \rightarrow B_{n}$ is a fiber bundle. Moreover, for each $n B_{n}=S^{1}$ 
and $E_{n}=M$ is a closed 3-manifold, all the $p_{n}$ 's are the same, and all the fibers of each $p_{n}$ are homeomorphic to the 2-torus $T^{2}$. The space $B$ is the dyadic solenoid.

Before proving Theorem 1 we first discuss some implications of it and its proof.

Corollary 1. There exists a shape fibration $p: E \rightarrow B$ with connected base-space $B$ such that $p$ has two fibers of different shape.

Corollary 1 follows immediately from Theorem 1 and answers a question posed in [8].

REMARK 1. One might at first assume that connectedness in shape theory is the natural analogue of path connectedness in homotopy theory. There are many instances when this is true, but not in general. Connectedness by shape paths (which is equivalent to pointed 1-movability [6]) seems to be the more accurate analogue. Theorem 1 and Corollary 1 support this thesis. Another argument supporting this thesis is the example by the first author of a metric continuum whose shape groups and homotopy pro-groups depend on the choice of base point [4]. Neither the example proving Theorem 1 in this paper nor the example in [4] can exist if the space is connected by shape paths.

REMaRK 2. Recently M. Jani [1] has defined a notion of fiber shape equivalence of shape fibrations. He has shown that the pullbacks of a shape fibration by strongly shape equivalent maps are fiber shape equivalent shape fibrations. Now any two maps of a point into a continuum $B$ are shape equivalent (but not necessarily strongly shape equivalent). However, the pull-backs in this case are just the fibers over the two image points. Consequently, Corollary 1 shows that one cannot strengthen Jani's result by replacing strong shape equivalence by shape equivalence.

REMARK 3. In a recent paper [7] the second author has introduced a new equivalence relation between metric compacta called $S$-type. It was easy to show that shape equivalent compacta have the same $S$-type and it was asked whether there were $S$-equivalent compacta of different shape. In [7] it was shown that for shape fibrations over connected spaces, the fibers have the same $S$-type. Consequently our theorem also has the following corollary.

CoROLLARY 2. There exist two 2-torus-like metric continua which have the same S-type, but which have different shape. 
In the proof of Theorem 1, it is shown that the two fibers of different shape actually have different 1-dimensional Čech cohomology groups. Thus we have the following corollary.

COROLlARY 3. The Čech cohomology groups with integer coefficients are not invariants of S-type.

REMARK 4. The fibers in our example are compact connected abelian topological groups. This was a definite aid in developing the example. The basic theory of shape for such groups was developed by the first author in [2]. Some other applications of the theory are given in [3] and [5].

2. Integral matrices and maps between tori. In the construction of the example $p: E \rightarrow B$ establishing Theorem 1 we shall be considering maps of the 2-torus $T^{2}$ into $T^{2}$. In this section we review briefly some facts concerning maps from the $n$-torus $T^{n}$ into $T^{n}$.

We think of $S^{1}$ as the multiplicative group of complex numbers of norm one. Then $T^{n}=S^{1} \times \cdots \times S^{1}$ becomes a compact connected abelian topological group. From [9] every continuous maps $f: T^{n} \rightarrow T^{n}$ is homotopic to a unique continuous homomorphism. Every homomorphism $f: T^{n} \rightarrow T^{n}$ can be represented by an $n \times n$ matrix with integer entries in the following manner. Let $e: R \rightarrow S^{1}$ be the standard covering may defined by $e(t)=e^{2 \pi i t}$. Then $e^{n}=e \times \cdots \times e$ : $R^{n} \rightarrow T^{n}$ is a homomorphism of topological groups. Every homomorphism $f: T^{n} \rightarrow T^{n}$ lifts to a unique homomorphism $\widetilde{f}: R^{n} \rightarrow R^{n}$ so that the following diagram commutes.

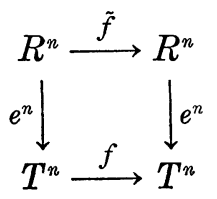

It follows that $\tilde{f}\left(\operatorname{ker} e^{n}\right) \subset \operatorname{ker} e^{n}$. But $\operatorname{ker} e^{n}=Z^{n}$ and thus $g=\widetilde{f} \mid Z^{n}$ is a homomorphism from $Z^{n}$ to $Z^{n}$. We associate with $f$ the unique $n \times n$ matrix $M$ such that $\left(y_{1}, \cdots, y_{n}\right)=g\left(x_{1}, \cdots, x_{n}\right)$ implies that

$$
M\left(\begin{array}{c}
x_{1} \\
\vdots \\
x_{n}
\end{array}\right)=\left(\begin{array}{c}
y_{1} \\
\vdots \\
y_{n}
\end{array}\right) .
$$

Clearly $M$ is an integral matrix. Conversely, if $M$ is any integral $n \times n$ matrix we obtain by (2) a continuous homomorphism $\widetilde{f}: R^{n} \rightarrow R^{n}$. Because $M$ is integral $\widetilde{f}\left(Z^{n}\right) \subset Z^{n}$. Therefore $\widetilde{f}$ defines 
a homomorphism $f$ on $T^{n}$ to $T^{n}$ so that (1) commutes. The homomorphism $f$ is unique.

Note that $f: T^{n} \rightarrow T^{n}$ is an isomorphism if and only if the associated matrix $M$ has $\operatorname{det} M= \pm 1$. Actually $|\operatorname{det} M|$ is the number of points in each point inverse set of $f$ if $\operatorname{det} M \neq 0$, but we shall not use this fact.

3. The construction of $p$. Let $g: T^{2} \rightarrow T^{2}$ be the continuous map determined by the integral matrix $\left(\begin{array}{ll}1 & 0 \\ 1 & 1\end{array}\right)$. Since the determinant of this matrix is one, $g$ is a homomorphism. Consider $T^{2} \times R$ and define an action of the group $Z$ on $T^{2} \times R$ by $n(x, t)=\left(g^{n}(x)\right.$, $t-n)$. Let $M$ be the quotient space of this action and let $r: T^{2} \times R \rightarrow M$ be the quotient map. Then $r$ is a covering map and $M$ is a closed 3-manifold. Note that $M=r\left(T^{2} \times[0,1]\right)$. Note also that $M$ can also be described as the mapping torus of $g: T^{2} \rightarrow T^{2}$. Let $\pi: T^{2} \times R \rightarrow R$ denote projection onto the second coordinate and let $e: R \rightarrow S^{1}$ denote the exponential map $t \rightarrow e^{2 \pi i t}$. Then there is a unique map $s: M \rightarrow S^{1}$ such that the following diagram commutes.

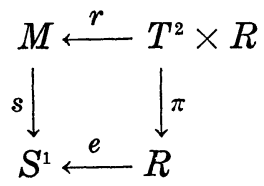

Indeed, if $r(x, t)=r\left(x^{\prime}, t^{\prime}\right)$, then there is an $n$ in $Z$ such that $\left(x^{\prime}, t^{\prime}\right)=\left(g^{n}(x), t-n\right)$. Hence, $e \pi\left(x^{\prime}, t^{\prime}\right)=e \pi\left(g^{n}(x), t-n\right)=e(t-n)=$ $e(t)=e \pi(x, t)$. Clearly, $s$ is a fiber bundle and thus a fibration.

Similarly, let $2: R \rightarrow R$ denote multiplication by 2 . Then there is a unique map $q: S^{1} \rightarrow S^{1}$ such that the following diagram commutes.

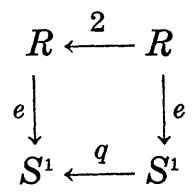

Of course, $q$ is just $z^{2}$ on the complex numbers of norm one.

Now let $f: T^{2} \rightarrow T^{2}$ be the continuous map induced by the matrix $\left(\begin{array}{ll}1 & 0 \\ 0 & 2\end{array}\right)$. We claim that there exists a unique map $h: M \rightarrow M$ such that the following diagram commutes.

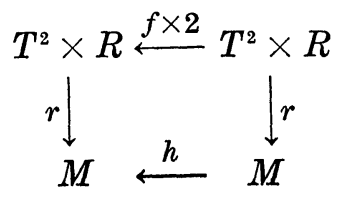


Moreover, $h$ makes the following diagram commutative also.

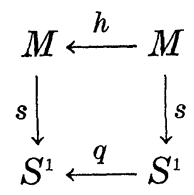

The last claim follows from the preceeding one because $r$ is onto and for $(x, t)$ in $T^{2} \times R$ we have by (1), (2), and (3) the following equalities.

$$
\begin{aligned}
q s r(x, t) & =q e \pi(x, t)=e(2 t)=e \pi(f(x), 2 t) \\
& =\operatorname{sr}(f(x), 2 t)=\operatorname{sr}(f \times 2)(x, t)=\operatorname{sh} r(x, t) .
\end{aligned}
$$

In order to prove the existence of $h$ we have to show that:

$$
r(x, t)=r\left(x^{\prime}, t^{\prime}\right)
$$

implies that

$$
r(f \times 2)(x, t)=r(f \times 2)\left(x^{\prime}, t^{\prime}\right) .
$$

Now (6) implies that there is an $n$ in $Z$ such that $x^{\prime}=g^{n}(x)$ and $t^{\prime}=t-n$. But then $r(f \times 2)\left(x^{\prime}, t^{\prime}\right)=r\left(f g^{n}(x), 2 t-2 n\right)$. It thus suffices to show that:

$$
f g^{n}=g^{2 n} f
$$

because then one has:

$$
\begin{aligned}
r(f \times 2)\left(x^{\prime}, t^{\prime}\right) & =r\left(f g^{n}(x), 2 t-2 n\right) \\
& =r\left(g^{2 n} f(x), 2 t-2 n\right) \\
& =r(f(x), 2 t) \\
& =r(f \times 2)(x, t) .
\end{aligned}
$$

The third equality in (9) holds because $2 n(f(x), 2 t)=\left(g^{2 n} f(x), 2 t-2 n\right)$. Thus we need to establish (8) to verify that $h$ is well-defined. But (8) is verified by the following matrix calculation.

$$
\begin{aligned}
& \left(\begin{array}{ll}
1 & 0 \\
0 & 2
\end{array}\right)\left(\begin{array}{ll}
1 & 0 \\
1 & 1
\end{array}\right)^{n}=\left(\begin{array}{ll}
1 & 0 \\
0 & 2
\end{array}\right)\left(\begin{array}{ll}
1 & 0 \\
n & 1
\end{array}\right)=\left(\begin{array}{rr}
1 & 0 \\
2 n & 2
\end{array}\right) \\
& \left(\begin{array}{ll}
1 & 0 \\
1 & 1
\end{array}\right)^{2 n}\left(\begin{array}{ll}
1 & 0 \\
0 & 2
\end{array}\right)=\left(\begin{array}{rr}
1 & 0 \\
2 n & 1
\end{array}\right)\left(\begin{array}{ll}
1 & 0 \\
0 & 2
\end{array}\right)=\left(\begin{array}{rr}
1 & 0 \\
2 n & 2
\end{array}\right) .
\end{aligned}
$$

Thus the matrices associated with $f g^{n}$ and $g^{2 n} f$ are the same and the maps $f g^{n}$ and $g^{2 n} f$ must coincide. Thus $h$ is well-defined.

We now define the inverse sequences $\underline{E}=\left(E_{n}, h_{n}\right)$ and $\underline{B}=\left(B_{n}, q_{n}\right)$ and the level map of fibrations $\underline{p}=\left(p_{n}\right)$. We let $E_{n}=M$ for all $n$ 
and $h_{n}=h$ for all $n$. We let $B_{n}=S^{1}$ for all $n$ and $q_{n}=q$ for all $n$. The we let $p_{n}=s$ for all $n$. Then $\underline{p}=\left(p_{n}\right)$ is a level map by (4) and since each $p_{n}$ is a fibration, $\underline{p}$ is a level map of fibrations. Finally, we let $E=\lim E, B=\lim B$, and $p=\lim \underline{p}$. The example has now been completely described. We need to show now that the example has the desired properties to prove Theorem 1 and its corollaries.

We observe that $B$ is clearly the dyadic solenoid. Moreover, all the fibers of $p_{n}$ are 2-tori from (1). This implies that the fibers of $p$ are inverse limits of 2-tori and are thus 2-torus-like continua. In [3] it is shown that the shape of a torus-like continuum is determined by its 1-dimensional Čech cohomology group. The only way two fibers $F$ and $F^{\prime}$ of $p$ can have different shape is if $H^{1}(F)$ and $H^{1}\left(F^{\prime}\right)$ are groups which are not isomorphic. In the next section of the paper we exhibit the fibers $F$ and $F^{\prime}$. Then in $\S 5$ we show that the fibers $F$ and $F^{\prime}$ are not shape equivalent by showing that $H^{1}(F)$ and $H^{1}\left(F^{\prime}\right)$ are not isomorphic groups.

4. Choosing the fibers $F$ and $F^{\prime}$. In this section we show how to choose the fibers $F$ and $F^{\prime}$ of $p$. For the construction of $F$ and $F^{\prime}$ we need two lemmas.

Lemma 1. Let $s_{n}: T^{2} \rightarrow T^{2}$ be a sequence of maps such that for each $n s_{n}=f$ or $s_{n}=g f$. Then there is a point $b$ in $B$ such that the fiber $F_{b}=p^{-1}(b)$ is homeomorphic to the inverse limit of the inverse sequence $\left(T^{2}, s_{n}\right)$.

Proof. We shall define points $b_{n}$ in $B_{n}$ and homeomorphisms $\mu_{n}$ so that

$$
q_{n}\left(b_{n+1}\right)=b_{n}
$$

and so that the following diagram commutes:

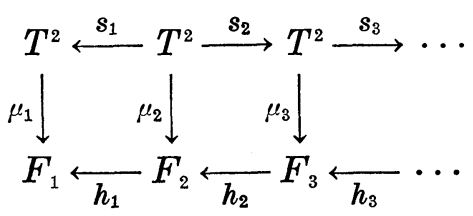

where $F_{i}=p_{i}^{-1}\left(b_{i}\right)$. Then letting $b=\lim \left(b_{n}\right)$ and $F_{b}=\lim \left(F_{n}, h_{n}\right)$ we will have the desired fiber. We will $\overleftarrow{\text { define }} b_{n}, F_{n}$, and the homeomorphisms $\mu_{n}$ by induction on $n$.

The points $b_{n}$ and the homeomorphism $\mu_{n}: T^{2} \rightarrow F_{n}$ will be defined by the formulas 


$$
b_{n}=e\left(t_{n}\right)
$$

and

$$
\mu_{n}(x)=r\left(x, t_{n}\right) \text { for } x \text { in } T^{2}
$$

where $t_{n}$ is in $R$ and will depend only on $n$.

Note that by (1) of $\S 3$

$$
p_{n} \mu_{n}(x)=\operatorname{sr}\left(x, t_{n}\right)=e \pi\left(x, t_{n}\right)=e\left(t_{n}\right)=b_{n}
$$

so that $\mu_{n}$ does map $T^{2}$ into $F_{n}=p_{n}^{-1}\left(b_{n}\right)$. Moreover, $\mu_{n}$ will be a homeomorphism. Indeed, let $y$ be in $F_{n}$. Then there is an $(x, t)$ in $T^{2} \times R$ such that $r(x, t)=y$ because $r$ is onto. Then by (1) of $\S 3$, $e(t)=e \pi(x, t)=s r(x, t)=s(y)=b_{n}$. Now there is a unique $m$ in $Z$ such that $r\left(g^{m}(x), t-m\right)=r(x, t)=y$ and $t-m=t_{n}$. Thus $x^{\prime}=$ $g^{m}(x) \in T^{2}$ is the only point such that $\mu_{n}\left(x^{\prime}\right)=y$.

We now show how to choose $t_{n}$ in $R$. We start the induction by putting $t_{1}=0$. Now assume that $t_{1}, \cdots, t_{k}$ have been defined so that (1) holds for $n=1,2, \cdots, k-1$ and the diagram (2) holds as far as it has been defined. We have to define $t_{k+1}$ anp verify (1) for $n=k$ and verify the commutativity of the following diagram.

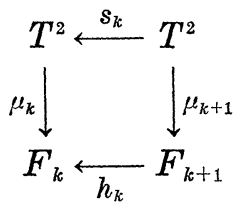

We distinguish two cases.

Case (i): Let $s_{k}=f$.

In this case we put $t_{k+1}=\frac{1}{2} t_{k}$. Then for $b_{k+1}=e\left(t_{k+1}\right)$ we have by $\S 3(2)$ :

$$
q_{k}\left(b_{k+1}\right)=q e\left(t_{k+\tau}\right)=q e\left(\frac{1}{2} t_{k}\right)=e 2\left(\frac{1}{2} t_{k}\right)=e\left(t_{k}\right)=b_{k} .
$$

Also, by $\S 3$ (3) we have:

$$
\begin{aligned}
h_{k} \mu_{k+1}(x) & =h r\left(x, t_{k+1}\right)=h r\left(x, \frac{1}{2} t_{k}\right)=r(f \times 2)\left(x, \frac{1}{2} t_{k}\right) \\
& =r\left(f(x), t_{k}\right)=\mu_{k}(f(x))=\mu_{k} s_{k}(x)
\end{aligned}
$$

as required by (6).

Case (ii): Let $s_{k}=g f$.

In this case we put $t_{k+1}=1 / 2\left(t_{k}+1\right)$. Then we have: 


$$
\begin{aligned}
q_{k}\left(b_{k+1}\right) & =q_{k} e\left(t_{k+1}\right)=q e\left(\frac{1}{2}\left(t_{k}+1\right)\right)=e\left(t_{k}+1\right) \\
& =e\left(t_{k}\right)=b_{k} .
\end{aligned}
$$

Furthermore,

$$
\begin{aligned}
h_{k} \mu_{k+1}(x) & =h r\left(x, t_{k+1}\right)=h r\left(x, \frac{1}{2}\left(t_{k}+1\right)\right) \\
& =r(f \times 2)\left(x, \frac{1}{2}\left(t_{k}+1\right)\right)=r\left(f(x), t_{k}+1\right) .
\end{aligned}
$$

Now by definition of $r$,

$$
r\left(f(x), t_{k}+1\right)=r\left(g f(x), t_{k}\right)=\mu_{k}(g f(x))=\mu_{k} s_{k}(x),
$$

as required by (6). This completes the proof.

We now define the fibers $F$ and $F^{\prime}$ using Lemma 1 so as to obtain the following lemma.

LEMMA 2. Let $1 \leqq n_{1}<n_{2}<n_{3}<\cdots$ be a sequence of integers. Then there exist points $b$ and $b^{\prime}$ in $B$ such that the fiber $F=p^{-1}(b)$ is homeomorphic to the limit of the inverse sequence of 2-tori $T^{2}$ with all bonding maps equal to $f$ and the fiber $F^{\prime}=p^{-1}\left(b^{\prime}\right)$ is homeomorphic to the limit of the inverse sequence of 2-tori where one has first $n_{1}$ bonding maps $g f$, then $n_{2}$ bonding maps $f$, then $n_{3}$ bonding maps $g f$, etc.

This is an immediate application of Lemma 1 . Lemma 2 now defines the fibers $F$ and $F^{\prime}$ which we claim are not shape equivalent. In the next two sections we show that $H^{1}(F)$ and $H^{1}\left(F^{\prime}\right)$ are not isomorphic which will prove our claim.

5. Cohomology of the fibers $F$ and $F^{\prime}$. Let $H^{1}$ denote the first Čech cohomology groups with coefficient group $Z$. Recall that there are natural isomorphisms $H^{1}\left(T^{2}\right) \cong \operatorname{Hom}\left(H_{1}\left(T^{2}\right), Z\right.$ ) (from the Universal Coefficient Theorem for cohomology) and $H_{1}\left(T^{2}\right) \cong \pi_{1}\left(T^{2}\right) \cong Z^{2}$ (since $\pi_{1}\left(T^{2}\right)$ is abelian). Thus one can identify $H^{1}\left(T^{2}\right)$ with $Z^{2}$. Now if one has a homomorphism $f: T^{2} \rightarrow T^{2}$ represented by the matrix $A$, then that homomorphism induces a homomorphism on $H^{1}\left(T^{2}\right)=Z^{2} \rightarrow Z^{2}=H^{1}\left(T^{2}\right)$. The induced homomorphism we can take to be represented by the transpose $A^{\prime}$ of the matrix $A$ if we make the following choice of basis for $H^{1}\left(T^{2}\right)$. Let $\left\{e_{1}, e_{2}\right\}$ be the basis for $\pi_{1}\left(T^{2}\right)$ corresponding to the basis in $Z^{2}$ by which the matrix $A$ was identified with $f$. Let $\left\{e_{1}^{\prime}, e_{2}^{\prime}\right\}$ be the basis in $H_{1}\left(T^{2}\right)$ under the 
natural transformation between $\pi_{1}\left(T^{2}\right)$ and $H_{1}\left(T^{2}\right)$. Then let $\left\{e_{1}^{\prime \prime}, e_{2}^{\prime \prime}\right\} \subset$ $\operatorname{Hom}\left(H_{1}\left(T^{2}\right), Z\right)$ be defined by $e_{i}^{\prime \prime}\left(e_{j}^{\prime}\right)=\delta_{i j}$ where $\delta_{i j}$ is the Kronecker delta. Then $\left\{e_{1}^{\prime \prime}, e_{2}^{\prime \prime}\right\}$ is a basis for $\operatorname{Hom}\left(H_{1}\left(T^{2}\right), Z\right)$. The image of this basis in $H^{1}\left(T^{2}\right)$ will be the required basis and with that basis $f^{*}: H^{1}\left(T^{2}\right) \rightarrow H^{1}\left(T^{2}\right)$ will be associated with the integral matrix $A^{\prime}$. These facts together with the continuity of the Čech cohomology groups implies that $H^{1}(F)$ is the direct limit of the direct sequence of groups:

$$
Z^{2} \stackrel{q_{1}}{\longrightarrow} Z^{2} \stackrel{q_{2}}{\longrightarrow} Z^{2} \stackrel{q_{3}}{\longrightarrow} \cdots
$$

where all the $q_{i}$ 's are given by the matrix $\left(\begin{array}{ll}1 & 0 \\ 0 & 2\end{array}\right)$.

Clearly the direct limit of this direct sequence of groups is isomorphic to $Z \oplus Q^{(2)}$ where $Q^{(2)}=\left\{m / 2^{n} \mid m, n \in Z, n \geqq 0\right\}$. Thus all that remains to be proved is that $H^{1}\left(F^{\prime}\right)$ is not isomorphic to $Z \oplus Q^{(2)}$. Now the matrix representing $g f$ is $\left(\begin{array}{ll}1 & 0 \\ 1 & 2\end{array}\right)$. Taking this fact together with the definition of $F^{\prime}$ into account, it will suffice to prove the following lemma.

LEMmA 3. Let $1 \leqq n_{1}<n_{2}<n_{3}<\cdots$ be a sequence of integers and let

$$
G_{0} \stackrel{q_{1}}{\longrightarrow} G_{1} \stackrel{q_{2}}{\longrightarrow} G_{2} \stackrel{q_{3}}{\longrightarrow} \cdots
$$

be a direct sequence of groups such that $G_{i}=Z^{2}$ for all $i$ such that the first $n_{1}$ bonding homomorphisms are given by matrix

$$
\left(\begin{array}{ll}
1 & 1 \\
0 & 2
\end{array}\right)
$$

the next $n_{2}$ bonding homomorphisms are given by

$$
F=\left(\begin{array}{ll}
1 & 0 \\
0 & 2
\end{array}\right)
$$

the next $n_{3}$ bonding homomorphisms are given by $H$, etc. Then the direct limit is a group $G$ which is not isomorphic to $Z \oplus Q^{(2)}$.

In $\S 6$ we prove Lemma 3 and thus complete the proof of Theorem 1.

6. Algebraic lemmas. We shall prove Lemma 3. That is, we shall show that the $G$ in Lemma 3 cannot be isomorphic to $Z \oplus Q^{(2)}$. This will be shown by showing that $G$ has a certain property $\alpha$, which is invariant under group isomorphism, which is not possessed 
by $Z \oplus Q^{(2)}$.

We say that an abelian group $A$ has property $\alpha$ provided there exists a monomorphism $\mu: A \rightarrow R^{2}$ and a sequence of elements $a_{i}$ in $A$ such that $\lim \mu\left(a_{i}\right)=0$ and such that any two of the elements $\mu\left(a_{i}\right)$ are linearly independent in $R^{2}$. Clearly, if $A$ has property $\alpha$ and $A \cong A^{\prime}$, then $A^{\prime}$ also has property $\alpha$. Thus Lemma 3 will follow from the next two lemmas.

Lemma 4. The group $G$ described in Lemma 3 has property $\alpha$.

Lemma 5. The group $Z \oplus Q^{(2)}$ fails to have property $\alpha$.

Proof of Lemma 4. We shall first define by induction a sequence of monomorphisms $\mu_{i}: Z^{2} \rightarrow R^{2}$ such that $\mu_{0}$ is the inclusion $G_{0}=Z^{2} \subset R^{2}$ and such that the following diagram commutes:

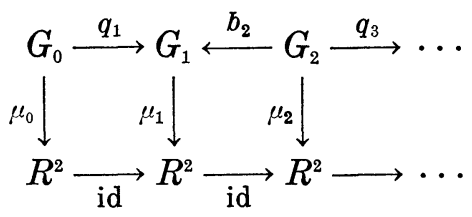

Let us assume that $\mu_{i-1}$ has already been defined. Since $\operatorname{det} F=$ $\operatorname{det} H=2 \neq 0, q_{i}$ is a monomorphism. Thus the subgroup $q_{i}\left(G_{i-1}\right)$ of $G_{i}$ is a free abelian group of rank 2. Thus there is a basis $\left\{a_{1}, a_{2}\right\}$ of $Z^{2}=G_{i}$ and integers $n_{1}, n_{2} \geqq 1$ such that $\left\{n_{1} a_{1}, n_{2} a_{2}\right\}$ is a basis for $q_{i}\left(G_{i-1}\right)$. Since $q_{i}: G_{i-1} \rightarrow q_{i}\left(G_{i-1}\right)$ is an isomorphism, there exists also a basis $\left\{e_{1}, e_{2}\right\}$ of $G_{i-1}$ such that

$$
q_{i}\left(e_{1}\right)=n_{1} a_{1} \text { and } q_{i}\left(e_{2}\right)=n_{2} a_{2} .
$$

Since $R^{2}$ is a divisible group one can find elements $b_{1}, b_{2}$ in $R^{2}$ such that

$$
n_{1} b_{1}=\mu_{i-1}\left(e_{1}\right) \text { and } n_{2} b_{2}=\mu_{i-1}\left(e_{2}\right) .
$$

Now define $\mu_{i}: G_{i}=Z^{2} \rightarrow R^{2}$ by putting

$$
\mu_{i}\left(a_{1}\right)=b_{1} \text { and } \mu_{i}\left(a_{2}\right)=b_{2} .
$$

Clearly, $\quad \mu_{i} q_{i}\left(e_{1}\right)=\mu_{i}\left(n_{1} a_{1}\right)=n_{1} \mu_{i}\left(a_{1}\right)=n_{1} b_{1}=\mu_{i-1}\left(e_{1}\right) . \quad$ Similarly, $\mu_{i} q_{i}\left(e_{2}\right)=\mu_{i-1}\left(e_{2}\right)$, so that induced $\mu_{i} q_{i}=\mu_{i-1}$. Now we show that $\mu_{i}$ is a monomorphism. If $k_{1}, k_{2}$ are in $Z$ and $\mu_{i}\left(k_{1} a_{1}+k_{2} a_{2}\right)=0$, then it follows that $k_{1} b_{1}+k_{2} b_{2}=0$ and therefore $\mu_{i-1}\left(n_{2} k_{1} e_{1}+n_{1} k_{2} e_{2}\right)=$ $n_{1} n_{2}\left(k_{1} b_{1}+k_{2} b_{2}\right)=0$. Since $\mu_{i-1}$ is a monomorphism, we conclude that $n_{2} k_{1} e_{1}+n_{1} k_{2} e_{2}=0$. However, $\left\{e_{1}, e_{2}\right\}$ is a basis for $Z^{2}$ so that $n_{2} k_{1}=$ $n_{1} k_{2}=0$. Thus $k_{1}=k_{2}=0$. 
It can be readily seen that the monomorphisms $\mu_{i}$ induce an isomorphism $\mu$ of the direct limit $G$ of $\left(G_{i}, q_{i}\right)$ onto the subgroup $B=\bigcup_{i=0}^{\infty} \mu_{i}\left(G_{i}\right) \subset R^{2}$.

We now consider the points $a_{i}$ in $G_{n_{1}+\cdots+n_{i}}=Z^{2}$ defined as follows.

$$
a_{i}=\left(\begin{array}{ll}
(1,1) & i \text { ood } \\
(0,1) & i \text { even }
\end{array}\right.
$$

Let

$$
b_{i}=\mu_{n_{1}+\cdots+n_{i}}\left(a_{i}\right), \quad b_{0}=(0,1) .
$$

We shall show that

$$
b_{i+1}=2^{-n_{i+1}}\left(b_{i}+(-1)^{i}(1,0)\right) .
$$

First assume that $i$ is even. Then the homomorphism $q_{n_{1}+\cdots+n_{i+1}} \circ \cdots \circ q_{n_{1}+\cdots+n_{i}+1}$ is given by the matrix $H^{n_{i+1}}$. Since

$$
H^{n_{i+1}}\left(\begin{array}{l}
1 \\
1
\end{array}\right)=2^{n_{i+1}}\left(\begin{array}{l}
1 \\
1
\end{array}\right)
$$

(1) implies that

$$
\begin{aligned}
2^{n_{i+1}} b_{i+1} & =\mu_{n_{1}+\cdots+n_{i+1}} 2^{n_{2+1}}(1,1) \\
& =\mu_{n_{1}+\cdots+n_{i}}(1,1) .
\end{aligned}
$$

Since

$$
F\left(\begin{array}{l}
1 \\
0
\end{array}\right)=H\left(\begin{array}{l}
1 \\
0
\end{array}\right)=\left(\begin{array}{l}
1 \\
0
\end{array}\right)
$$

it follows that

$$
\begin{aligned}
\mu_{n_{1}+\cdots+n_{i}}(1,1) & =(1,0)+\mu_{n_{1}+\cdots+n_{i}}(0,1) \\
& =(1,0)+b_{i} .
\end{aligned}
$$

Now (9) and (11) yield (7) for even $i$.

Using the fact that

$$
F^{n_{i+1}}\left(\begin{array}{l}
0 \\
1
\end{array}\right)=2^{n_{i+1}}\left(\begin{array}{l}
0 \\
1
\end{array}\right)
$$

one can verify (7) for odd $i$ in a similar way.

Let $b_{i}=\left(x_{i}, y_{i}\right)$ and let $s_{k}=\sum_{j=1}^{k} n_{j}$. From the recursive formula (7) it is easy to derive the formulas.

$$
x_{i}=2^{-s_{i}}\left(1+\sum_{j=1}^{i-1}(-1)^{j} 2^{s_{j}}\right)
$$




$$
y_{i}=2^{-s_{i}} \text {. }
$$

Now we shall show that $b_{n}$ and $b_{m}$ are linearly independent in $R^{2}$ for $n<m$. Indeed, assume that

$$
\lambda b_{n}+\mu b_{m}=0 \quad n<m .
$$

Then (14) yields

$$
\lambda 2^{-s_{n}}+\mu 2^{-s_{m}}=0 .
$$

The analogous relation for the first coordinates simplifies using (16) to the following.

$$
\lambda 2^{-s_{n}} \sum_{j=1}^{n-1}(-1)^{j} 2^{s_{j}}+\mu 2^{-s_{m}} \sum_{j=1}^{m-1}(-1)^{j} 2^{s_{j}}=0 .
$$

Now (16) and (17) yields:

$$
\mu 2^{-s_{m}} \sum_{j=n}^{m-1}(-1)^{j} 2^{s_{j}}=0 .
$$

The number

$$
\sum_{j=n}^{m-1}(-1)^{j} 2^{s_{j}}=2^{s_{n}}\left((-1)^{n}+\sum_{j=n+1}^{m-1}(-1)^{j^{j} 2^{s_{j}-s_{n}}}\right)
$$

is $\neq 0$ because the expression in the parentheses is an odd number. Consequently, (18) yields $\mu=0$. Then (16) yields $\lambda=0$.

Finally, to show that $\lim b_{i}=0$ it suffices to show that

$$
\left|b_{i}\right| \leqq 2 \cdot 2^{-n_{i}}
$$

This holds for $i=1$ because $b_{1}=2^{-n_{1}}(1,1)$.

If we assume (19) for $i$, then by (7)

$$
\left|b_{i+1}\right| \leqq 2^{-n_{i+1}}\left(\left|b_{i}\right|+1\right) \leqq 2 \cdot 2^{-n_{i+1}} \text {. }
$$

This completes the proof of Lemma 4 .

Proof of Lemma 5. Let $u: Z \oplus Q^{(2)} \rightarrow R^{2}$ be any monomorphism. One readily sees that $u$ extends to a linear map $v: R^{2} \rightarrow R^{2}$. Therefore $v(0 \times R)$ is a straight line $L \subset R^{2}$ through 0 . Also, $v(n \times R)$, $n \in Z$, all coincide with $L$ or are straight lines parallel to $L$ such that the distance between any two consecutive lines is the same number $r>0$. The first case is trivial. In the second case let $U$ denote the open disc around 0 of radius $r$, then $U \cap u\left(Z \oplus Q^{(2)}\right) \subset L$. Consequently, all pairs of points of $u\left(Z \oplus Q^{(2)}\right)$ which are close enough to 0 must be linearly dependent. Hence the group $Z \oplus Q^{(2)}$ fails to have property $\alpha$. 
This completes the proof of Lemma 5 and thus completes the proof of Theorem 1.

Added in proof. K. R. Goodearl and T. B. Rushing have recently shown that there are uncountably many fibers of different shape type in the example of the paper. Details are in "Direct limit groups and the Keesling-Mardesic shape fibration" (to appear in Pacific J. Math.).

\section{REFERENCES}

1. M. Jani, Induced shape fibrations and fiber shape equivalences, Ph. D. Thesis, CUNY, New York, 1978.

2. J. Keesling, Shape theory and compact connected abelian topological groups, Trans. Amer. Math. Soc., 194 (1974), 349-358.

3. On the shape of torus-like continua and compact connected topological groups. Proc. Amer. Math. Soc., 40 (1973), 297-302.

4. - Products in the shape category and some applications, Symposia Mathematica, 16 (1975), 133-142.

5. - Some examples in shape theory using the theory of compact connected abelian topological groups, Trans. Amer. Math. Soc., 219 (1976), 169-188.

6. J. Krasinkiewicz and P. Minc, Generalized paths and pointed 1-movability, Fund. Math., (to appear).

7. S. Mardešić, Comparing fibres of a shape fibration, Glasnik Mat., (to appear).

8. S. Mardešic and T.B. Rushing, Shape fibrations I, General Topology and Appl., (to appear).

9. W. Scheffer, Maps between topological groups that are homotopic to homomorphisms, Proc. Amer. Math. Soc., 33 (1972), 562-567.

Received October 3, 1978 and in revised form January 17, 1979. Work on this paper was initiated during a visit of the second author at the University of Florida, Gainesville, in May 1978.

UNIVERSITY OF FLORIDA

Gainesville, Fl 32611

AND

UNIVERSITY OF ZAGREB

Zagreb, Yugoslavia 



\section{PACIFIC JOURNAL OF MATHEMATICS}

\section{EDITORS}

DONALD BABBITT (Managing Editor)

University of California

Los Angeles, CA 90024

HUGO RossI

University of Utah

Salt Lake City, UT 84112

C. C. Moore and ANDrew OGG

University of California

Berkeley, CA 94720
J. DUGUNDJI

Department of Mathematics University of Southern California Los Angeles, CA 90007

R. FINN and J. MILGRAM

Stanford University

Stanford, CA 94305

\section{ASSOCIATE EDITORS}
E. F. BeCKENBACH
B. H. NeumanN
F. WOLF
K. YOSHIDA

\section{SUPPORTING INSTITUTIONS}

UNIVERSITY OF BRITISH COLUMBIA CALIFORNIA INSTITUTE OF TECHNOLOGY UNIVERSITY OF CALIFORNIA MONTANA STATE UNIVERSITY UNIVERSITY OF NEVADA, RENO NEW MEXICO STATE UNIVERSITY OREGON STATE UNIVERSITY UNIVERSITY OF OREGON

\author{
UNIVERSITY OF SOUTHERN CALIFORNIA \\ STANFORD UNIVERSITY \\ UNIVERSITY OF HAWAII \\ UNIVERSITY OF TOKYO \\ UNIVERSITY OF UTAH \\ WASHINGTON STATE UNIVERSITY \\ UNIVERSITY OF · WASHINGTON
}

The Supporting Institutions listed above contribute to the cost of publication of this Journal, but they are not owners or publishers and have no responsibility for its content or policies.

Mathematical papers intended for publication in the Pacific Journal of Mathematics should be in typed form or offset-reproduced, (not dittoed), double spaced with large margins. Please do not use built up fractions in the text of the manuscript. However, you may use them in the displayed equations. Underline Greek letters in red, German in green, and script in blue. The first paragraph or two must be capable of being used separately as a synopsis of the entire paper. Please propose a heading for the odd numbered pages of less than 35 characters. Manuscripts, in triplicate, may be sent to any one of the editors. Please classify according to the scheme of Math. Reviews, Index to Vol. 39. Supply name and address of author to whom proofs should be sent. All other communications should be addressed to the managing editor, or Elaine Barth, University of California, Los Angeles, California, 90024.

50 reprints to each author are provided free for each article, only if page charges have been substantially paid. Additional copies may be obtained at cost in multiples of 50 .

The Pacific Journal of Mathematics is issued monthly as of January 1966. Regular subscription rate: $\$ 84.00$ a year (6 Vols., 12 issues). Special rate: $\$ 42.00$ a year to individual members of supporting institutions.

Subscriptions, orders for numbers issued in the last three calendar years, and changes of address should be sent to Pacific Journal of Mathematics, P.O. Box 969, Carmel Valley, CA 93924, U.S.A. Older back numbers obtainable from Kraus Periodicals Co., Route 100, Millwood, NY 10546.

PUBLISHED BY PACIFIC JOURNAL OF MATHEMATICS, A NON-PROFIT CORPORATION

Printed at Kokusai Bunken Insatsusha (International Academic Printing Co., Ltd.). 8-8, 3-chome, Takadanobaba, Shinjuku-ku, Tokyo 160, Japan.

Copyright (C) 1979 by Pacific Journal of Mathematics Manufactured and first issued in Japan 


\section{Pacific Journal of Mathematics}

\section{Vol. 84, No. $2 \quad$ June, 1979}

Somesh Chandra Bagchi and Alladi Sitaram, Spherical mean periodic

functions on semisimple Lie groups ........................ 241

Billy Joe Ball, Quasicompactifications and shape theory............. 251

Maureen A. Bardwell, The o-primitive components of a regular ordered permutation group ................................ 261

Peter W. Bates and James R. Ward, Periodic solutions of higher order

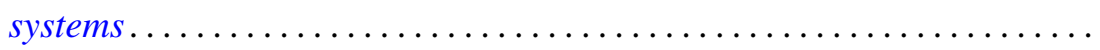

Jeroen Bruijning, A characterization of dimension of topological spaces by totally bounded pseudometrics......................... 283

Thomas Farmer, On the reduction of certain degenerate principal series

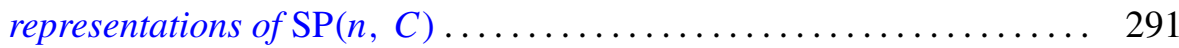

Richard P. Jerrard and Mark D. Meyerson, Homotopy with m-functions . . . . 305

James Edgar Keesling and Sibe Mardesic, A shape fibration with fibers of

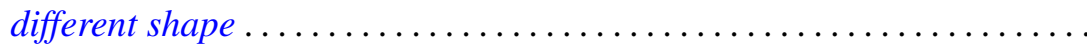

Guy Loupias, Cohomology over Banach crossed products. Application to bounded derivations and crossed homomorphisms ...............

Rainer Löwen, Symmetric planes ........................ 367

Alan L. T. Paterson, Amenable groups for which every topological left

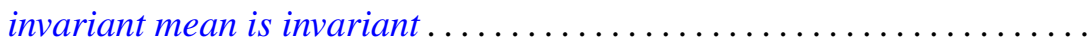

Jack Ray Porter and R. Grant Woods, Ultra-Hausdorff H-closed extensions

Calvin R. Putnam, Operators satisfying a $G_{1}$ condition .

Melvin Gordon Rothenberg and Jonathan David Sondow, Nonlinear smooth representations of compact Lie groups..............

Werner Rupp, Riesz-presentation of additive and $\sigma$-additive set-valued measures.

A. M. Russell, A commutative Banach algebra of functions of generalized variation

Judith D. Sally, Superregular sequences

Patrick Shanahan, On the signature of Grassmannians . . 